\title{
Círculo virtuoso de la cooperación e innovación en la pequeña y mediana empresa
}

\author{
De Pablo Valenciano, Jaime* \\ Uribe Toril, Juan**
}

\section{Resumen}

El objetivo de este artículo es explicar cómo la innovación y la cooperación se implementan en las pequeña y medianas empresas exitosas dentro de un entorno globalizado. A partir de la metodología de la ruta crítica, se inicia una descripción de secuencias de actividades empresariales: alianzas de cooperación, innovación (desde su perspectiva conceptual) y sinergia de la cooperación entre empresas. Una vez establecido el entorno conceptual del binomio cooperación-innovación, se conecta con el principio de asimetría de Steindl (1945), formador de redes de cooperación y de aglomeraciones empresariales (distritos industriales). Como principal conclusión de este camino, se demostrará la existencia de en un círculo virtuoso de cooperación-innovación empresarial.

Palabras clave: Cooperación; innovación; emprendedores; pequeñas y medianas empresas.

\section{Virtuous circle of the cooperation and innovation in the small and medium-sized enterprise}

\begin{abstract}
The aim of this study was to explain how the innovation and cooperation are implemented in successful small and medium-sized enterprises within a globalized environment. From the methodology of the critical path, starts a description of business activities sequences: cooperation alliances, innovation (from its conceptual perspective) and cooperation between enterprises synergy. Once established the binomial cooperation-innovation conceptual environment, it connects with the Steindls asymmetry principle (1945), maker of cooperation networks and business agglomerations (industrial districts). As main conclusion of this path, the existence a business cooperation-innovation virtuous circle will be demonstrated.
\end{abstract}

Key Words: Cooperation; innovation; entrepreneurs; small and medium-sized enterprises

Recibido: 20.04.13. Aceptado: 20.03.15

* Ph.D. Profesor Titular en la Facultad de CC. Económicas y Empresariales de la Universidad de Almería. Director de la Cátedra Iberoamericana de PRL, Negociación Colectiva de la UAL. E-mail:jdepablo@ual.es

** Ph.D. Profesor Asociado en la Facultad de CC. Económicas y Empresariales de la Universidad de Almería. E-mail:juribe@ual.es 


\section{Introducción}

Si se entiende la cooperación como "obrar juntamente con otro u otros para un mismo fin", se puede afirmar, sin ningún tipo de duda, que la cooperación empresarial existe desde el mismo nacimiento de la primera transacción mercantil. La empresa completamente autárquica, sin relación alguna con otras empresas de su entorno, es prácticamente imposible hasta de imaginar.

Cuando la empresa comienza su actividad, son múltiples los lazos, formales e informales, que se establecen. La finalidad de todas estas relaciones es la de obtener una situación beneficiosa frente al entorno, que permita un mayor flujo de transacciones comerciales, y a la postre, un mayor beneficio.

Estos lazos de cooperación se establecen de una forma constante, habitual y ágil, creando redes de empresas cuyo lazo de unión es la interacción y que no necesariamente necesitan estar en el mismo territorio aunque generalmente, el empresario de una manera consciente o inconsciente busca la concentración física de sus actividades (tanto para aminorar costos en sus producciones, como para aumentar su capacidad de comercio).

Un aliciente más hacia los movimientos de cooperación empresarial es la búsqueda de innovación, ya sea en productos, procesos, organización o comercialización, que a su vez propician entornos proclives a innovar, cerrándose un círculo virtuoso.

No es preciso que estas relaciones se articulen necesariamente en procedimientos formales o que de estos contactos se deriven en fusiones o dependencias, se contempla la cooperación desde un prisma amplio, sobre todo para la pequeña y mediana empresa (pyme), que posee una mayor flexibilidad en sus actuaciones y menores cargas estructurales jerárquicas en la toma de decisiones.

El objetivo de este artículo se centra en explicar cómo desde la innovación y la cooperación se pone en funcionamiento empresas exitosas dentro de un contexto marcado por la globalización. Para tal fin se analizan y discuten diversas posturas teóricas que abordan las variables en estudio (innovación y cooperación).

\section{Cooperación, innovación y pymes: factores intangibles}

Los factores intangibles se pueden definir como activos sin sustancia física, cuyos beneficios económicos futuros pueden ser controlados por la empresa y consisten, básicamente, en conocimiento o información. No tienen una entidad material y no son, por tanto, susceptibles de tocarse o percibirse de un modo preciso, por lo que se hace difícil su identificación y cuantificación (Hidalgo et al, 2002:505)

Tienen una influencia importante sobre la competitividad y los resultados de la empresa ya que sus características específicas les hacen tener un fuerte potencial diferenciador respecto de los competidores (Fernández et al, 1998: 96) pero requiere que la gestión empresarial se plantee con un horizonte temporal dilatado, y exige cambios importantes (mayor implicación y participación de los trabajadores) y formas organizativas flexibles y complejas (como los acuerdos de cooperación).

Los recursos intangibles presentan una serie de características específicas, entre las que se destacan (Navas y Guerras, 1998:17): 
$=$ - Invisibilidad: Al estar basados en la información y el conocimiento, no tienen soporte físico lo cual incrementa la dificultad de su tratamiento.

= - Dificultad de cuantificación: Por su propia naturaleza y composición, ya que no es medible ni codificable.

= Falta de aparición en los estados contables ${ }^{1}$ : Los estados financieros proporcionados por la contabilidad sólo recogen los activos tangibles, no considerando, salvo casos excepcionales, el valor de los intangibles.

- Apreciación por el uso: Ya que el conocimiento se basa en conocimiento, se introduce en la empresa la ley de rendimientos crecientes, complicando de nuevo la valoración contable de los mismos.

= - Lentitud de acumulación: Compuesto por la sedimentación de conocimientos e innovaciones en la empresa a lo largo de su historia.

Se pueden distinguir dos clases de intangibles.- personales e impersonales (Fernández, 2002:193) que se pueden subdividir a su vez en cuatro clases de recursos intangibles: capital humano, capital organizativo, capital tecnológico y la reputación (Fernández et al, 1999b:86). Los dos primeros pertenecen (capital humano y capital organizativo), al intangible personal, y los dos últimos (capital tecnológico y reputación) al intangible impersonal.

Las alianzas y cooperaciones entre empresas son elementos intangibles que pueden acontecer en cualquier área funcional de la empresa, siendo la alianza tecnológica o enfocada a componentes de innovación ${ }^{2}$ la que genera sinergias empresariales que suponen autenticas ventajas competitivas.

Para competir en un mundo como el actual es preciso innovar con una rapidez extraordinaria mejorando de forma continua los productos, servicios y procesos. Para ello, es necesario un nuevo y flexible modelo de empresa que sea capaz de liberar el ingenio humano y adaptarse a las cambiantes condiciones ambientales.

Los acuerdos de cooperación entre pequeñas y medianas empresas aparecen como una alternativa estratégica muy interesante para mejorar la competitividad de las mismas, al permitirles el acceso a mayores recursos, sin llegar a fusionarse, y por tanto manteniendo la flexibilidad inherente a ellas, que les facilita su adaptación a los cambios que se suceden en el entorno (Lapiedra, 1999:164).

Una concepción de innovación abierta sólo tiene cabida en una entidad donde exista una cultura fuerte y atrevida que dé la espalda a modelos rígidos.

En etapas pasadas, las innovaciones empresariales, sobre todo

1 El principio de prudencia contable aconseja no incorporar los intangibles en los balances de situación para no alterar inadecuadamente el valor de la empresa, debido precisamente a las dificultades de cuantificación y valoración, antes analizadas. Sólo en momentos concretos de la empresa, por ejemplo, en procesos de venta o fusión empresarial, aparece recogido el valor de los intangibles a través de la consideración del "good-will" o Fondo de Comercio.

2 Según el Manual de Oslo (OCDE, 2005) la innovación es: "La implementación de un producto (bien o servicio) o proceso nuevo o con un alto grado de mejora, o un método de comercialización u organización nuevo aplicado a las prácticas de negocio, al lugar de trabajo o a las relaciones externas". 
las de carácter tecnológico, se han considerado unidas al territorio como un mero soporte o escenario en el que discurrían los acontecimientos, pero se entiende ahora que es precisamente la capacidad innovadora entendida en un sentido más amplio -como la predisposición para incorporar conocimiento- la que, al permitir utilizar racionalmente los recursos patrimoniales existentes en cada ámbito es capaz de crear un entorno que propicia el desarrollo (Caravaca et al, 2003:104).

Pero no todas las regiones o todas las empresas tienen la misma capacidad para asimilar las trasformaciones acometidas por innovación en beneficios empresariales, siendo generalmente los factores sociales las claves de éxito o de fracaso de las políticas de desarrollo en innovación (Rodríguez-Pose, 1999:100).

Uno de los objetivos esenciales de cualquier organización es innovar para posicionarse mejor en su mercado. A medida que pasa el tiempo, todas las empresas tienen una mayor facilidad de acceso a todas las fuentes de información y conocimiento, la eficiencia dependerá de cómo se utilice este recurso (Morcillo, 2007:136).

Partiendo del Manual de Oslo de 2005 y la compilación que del mismo efectúa el profesor Frías (2006:25), se desglosan las siguientes teorias sobre innovación:

- Teoría de Schumpeter: ha influido extraordinariamente en las teorías de la innovación. El desarrollo económico está impulsado por la innovación mediante un proceso dinámico, en el cual las nuevas tecnologías sustituyen a las viejas en lo que se denomina "destrucción creativa". Las innovaciones radicales generan cambios o alteraciones importantes, requieren la combinación de piezas de conocimiento previamente desconectadas a diferencia de las incrementales que avanzan continuamente en el proceso de cambio y que están basadas en nuevas distinciones conceptuales, o nuevas maneras de combinar, elementos que puede que estén ya previamente asociados. Se plantean cinco tipos de innovación: introducción de productos nuevos; introducción de nuevos métodos de producción; apertura de nuevos mercados; desarrollo de nuevas fuentes de abastecimiento para las materias primas y creación de nuevas estructuras de mercado sectoriales.

- Teoría neoclásica: contempla la innovación en términos de creación de activos, así como de experimentos de mercado. En este enfoque, la innovación es un aspecto de la estrategia empresarial, o parte del sistema de decisiones de inversión, con el objetivo de crear la capacidad para el desarrollo de productos o para la mejora de la eficacia.

- Teoría evolutiva (Nelson, 1993: 56): se concibe la innovación como un proceso de trayectoria dependiente, en el cual se desarrollan el conocimiento y la tecnología mediante la interacción entre los diversos agentes y otros factores. La estructura de esta interacción afecta al itinerario futuro del cambio económico

- La innovación como sistema: subraya la importancia de la transferencia y difusión de ideas, cualificaciones, conocimiento, información y otras señales, yes una teoría estrechamente ligada al enfoque evolutivo. Los sistemas de innovación estudian la influencia de las instituciones externas en las actividades innovadoras de las empresas y de otros agentes 
(Lundvall, 1992:98; Nelson, 1993:34).

- Teoría de la organización industrial: se subraya el significado de la posición competitiva. Las empresas innovan para defender tanto su posición como para buscar nuevas ventajas competitivas.

- Otras líneas: se centran en el papel de las estructuras organizativas, de los procesos de aprendizaje y de la adaptación a los cambios en la tecnología y el entorno institucional $y$ de los mercados o en los factores que afectan a las decisiones de las empresas para adoptar nuevas tecnologías, su acceso a nuevos conocimientos y su capacidad de absorción.

La difusión de nuevos conocimientos y tecnologías ocupa un lugar central en la innovación y su proceso. El conocimiento proviene de fuentes tanto internas como externas a la organización, como son las externalidades positivas que las empresas reciben en términos de know how del entorno en el cual ellas operan. Particularmente, las relaciones inter organizativas pueden crear oportunidades para la adquisición y explotación de conocimiento.

El manual de Oslo de la OCDE (2005) señala cuatro tipos de innovación: - Innovación de producto: Supone alteraciones significativas en las características de las mercancías o de los servicios. Se incluyen tanto las mercancías totalmente nuevas como los servicios y las mejoras sustanciales de los productos ya existentes.

- Innovación de proceso: Debe basarse en cambios significativos en los métodos de producción y de distribución.

- Innovación de organización: Referida tanto a las relaciones exteriores de la empresa como a la puesta en práctica de nuevos métodos de trabajo, tanto de la organización como del lugar de trabajo.

- Innovación de comercialización: Que refleja la puesta en práctica de nuevos métodos de comercialización; desde cambios en el diseño y el empaquetado hasta la promoción del producto mediante nuevas políticas de precios y de servicios.

El comportamiento abierto frente a las innovaciones es, sin duda alguna, una muestra de fortaleza y valentía por parte de las empresas que asumen el reto de incorporar constantemente nuevos conocimientos y revela, además un carácter positivo (Morcillo, 2007:278).

La innovación es cuestión de actitudes (Sainz de Vicuña, 2006:89). Las capacidades de las empresas para innovar, se pueden asociar con la actitud para combinar e intercambiar recursos de conocimiento. Esa mentalidad abierta para el intercambio es la que propicia el encuentro con otras sociedades de similar espíritu y el inicio de la cooperación.

El conocimiento y la capacidad de organización que se desarrollan localmente, junto con la habilidad de integrarlas con los conocimientos que se derivan del progreso general de la tecnología, bien sea de base o bien sea específica orientada a la realización de un determinado producto, se propagan entre las empresas en cuanto miembros de una comunidad local, que comparten un mismo sistema de reglas y de costumbres que permiten el aprendizaje colectivo de conocimientos y de organización (Sforzi, 2002:247).

Se han recogido y resumido algunas de las clasificaciones sobre innovación, que se consideran importantes, bien por su generalidad, por su originalidad 
o por su utilidad en el campo de la investigación, basándonos en el estudio de Gisbert (2005:102) sobre creatividad e innovación (Cuadro 1).

\section{Cuadro 1}

\section{Clasificación de la estrategia de innovación}

\begin{tabular}{|c|c|c|}
\hline Clasificación & Modalidad & Descripción \\
\hline \multirow{5}{*}{$\begin{array}{l}\text { Por su objeto o } \\
\text { naturaleza }\end{array}$} & Tecnológica & $\begin{array}{l}\text { El cambio radica en el empleo de una nueva tecnología, } \\
\text { no experimentada o aplicada }\end{array}$ \\
\hline & Comercial & $\begin{array}{l}\text { Nueva presentación del producto, de su forma de } \\
\text { distribución, aplicación... }\end{array}$ \\
\hline & Organizativa & Nueva estructura, nuevo método de trabajo... \\
\hline & En proceso & Se introduce por primera vez en el mercado un proceso \\
\hline & En producto & $\begin{array}{l}\text { Introducción por primera vez en el mercado a través de } \\
\text { un producto }\end{array}$ \\
\hline \multirow{5}{*}{$\begin{array}{l}\text { Por su } \\
\text { inmaterialidad }\end{array}$} & Institucional & $\begin{array}{l}\text { Cambios de normas o sistemas nuevos introducidos por } \\
\text { los poderes públicos. }\end{array}$ \\
\hline & $\begin{array}{l}\text { Innovación } \\
\text { valorización }\end{array}$ & $\begin{array}{l}\text { Utilizar y aprovechar un conjunto de conocimientos y } \\
\text { experiencias para crear soluciones inéditas. En ocasiones } \\
\text { coproducida por el cliente y reconocida a posteriori. }\end{array}$ \\
\hline & $\begin{array}{l}\text { Innovación } \\
\text { anticipación }\end{array}$ & $\begin{array}{l}\text { Surge por la acción de vigilancia y atención del entorno, } \\
\text { detectando los problemas y necesidades nuevas de } \\
\text { los clientes, a través de un proceso de recolección y } \\
\text { acumulación de conocimientos y experiencias. }\end{array}$ \\
\hline & $\begin{array}{l}\text { Innovación } \\
\text { objetivación }\end{array}$ & $\begin{array}{l}\text { Dar materialidad al servicio por diferentes medios: } \\
\text { especificación formal de etapas, creación de una } \\
\text { metodología propia, incorporación de herramientas } \\
\text { creadas al efecto... }\end{array}$ \\
\hline & Emergente & Se generan en la organización como respuesta inédita \\
\hline \multirow{5}{*}{ Por su origen } & Adoptadas & Importadas desde fuera de la organización \\
\hline & Impuestas & Forzadas por exigencias externas o del mercado. \\
\hline & $\begin{array}{l}\text { Tirón de la } \\
\text { demanda }\end{array}$ & Tienden a cubrir una necesidad de la demanda \\
\hline & Empuje científico & Procedentes del propio avance de la ciencia \\
\hline & $\begin{array}{l}\text { Empuje } \\
\text { emprendedor }\end{array}$ & Procedente de aspectos personales del empresario \\
\hline
\end{tabular}

Fuente: Gisbert (2005). 


\section{Cont... Cuadro 1}

\begin{tabular}{|c|c|c|}
\hline Clasificación & Modalidad & Descripción \\
\hline \multirow{4}{*}{$\begin{array}{l}\text { Según el nivel } \\
\text { de cambio }\end{array}$} & Sintonización & Cambios incrementales anticipadores. \\
\hline & Adaptación & Incremental y reactivo. \\
\hline & Reorganización & Estratégico y anticipador. \\
\hline & Recreación & Estratégico y reactivo. \\
\hline \multirow{5}{*}{$\begin{array}{l}\text { Por su grado de } \\
\text { innovación }\end{array}$} & Radical & $\begin{array}{l}\text { Revolucionaria, a través de nueva tecnología, nuevos } \\
\text { materiales... }\end{array}$ \\
\hline & Incremental & $\begin{array}{l}\text { Explotación sistemática de conocimientos que permiten } \\
\text { introducir mejoras graduales }\end{array}$ \\
\hline & Gradual aditiva & Se trata de añadir algo nuevo a elementos antiguos. \\
\hline & $\begin{array}{l}\text { Gradual } \\
\text { sustitutiva }\end{array}$ & $\begin{array}{l}\text { Creación de productos, tecnologías o procesos nuevos } \\
\text { que reemplacen a las antiguos }\end{array}$ \\
\hline & Adaptada & $\begin{array}{l}\text { Innovación por imitación. Introducción en un mercado } \\
\text { específico de un producto copiado de una empresa } \\
\text { exterior. }\end{array}$ \\
\hline \multirow{4}{*}{$\begin{array}{l}\text { Innovación en } \\
\text { procesos }\end{array}$} & Simbólicos & $\begin{array}{l}\text { Incrementales y de poca importancia, denotan una } \\
\text { intencionalidad oculta. }\end{array}$ \\
\hline & Puntuales & Incrementales, resolución de problemas concretos. \\
\hline & Estructurales & Dirigidos a la estructura organizativa. \\
\hline & Transformadores & Dirigidos a la conducta y actitudes de las personas. \\
\hline \multirow{3}{*}{$\begin{array}{l}\text { Según la } \\
\text { magnitud de } \\
\text { cambio }\end{array}$} & Steamlining & $\begin{array}{l}\text { Mejora a través de nuevos sistemas y técnicas de } \\
\text { producción más eficientes. }\end{array}$ \\
\hline & Reingeniería & Cambio horizontal de algunos procesos \\
\hline & Transformación & Reingeniería aplicada a toda la empresa \\
\hline \multirow{8}{*}{$\begin{array}{l}\text { Innovación } \\
\text { según su } \\
\text { génesis } \\
\text { motivadora }\end{array}$} & Replicante & Copia para mantener una posición \\
\hline & Redefinitoria & Cambio de la perspectiva de la acción \\
\hline & Hacia la mejora & Cambio hacia estrategias nuevas pero ya experimentadas \\
\hline & $\begin{array}{l}\text { Mejora } \\
\text { avanzada }\end{array}$ & Cambio hacia estrategias nuevas y no experimentadas \\
\hline & Redirección & Se introduce un cambio en la dirección \\
\hline & Reconstrucción & Se vuelve a una situación pasada para afrontar cambios \\
\hline & Reiniciación & Vuelta al inicio para reconducir el cambio \\
\hline & Integración & $\begin{array}{l}\text { Combinación de distintas fuentes para afrontar el cambio } \\
\text { o innovación }\end{array}$ \\
\hline
\end{tabular}

Fuente: Gisbert (2005). 
Si bien la innovación es la búsqueda de lo nuevo, también se afirma que no existe nada completamente nuevo. El conocimiento humano se basa en experiencias y asociaciones, siempre se puede llegar a un antecedente de la invención.

Estas afirmaciones, un tanto engañosas, sirven para abrir la mente y pensar que la innovación empresarial no suele corresponder a grandes adelantos surgidos de la nada, sino que son pequeñas modificaciones o alteraciones de algo, pero que introducen alguna novedad. Por tanto, con una concepción amplia, innovación es cualquier modo de "hacer las cosas de modo distinto" en el reino de la vida económica, todo son modalidades de lo que se denomina innovación (Shumpeter, 1939:2).

La innovación es un proceso complejo y con múltiples implicaciones, en el cual confluyen conocimientos científicos y tecnológicos junto a otros sociales, de marketing y creativos, y cuyo éxito o fracaso van a estar determinados por las estructuras organizativas de las empresas e instituciones que se proponen desarrollar el proceso, por las características de los empresarios y directivos, por la necesidades, y por la aceptación por parte del mercado y de la sociedad del cambio introducido (Ondategui, 2001:24). Es un proceso sistemático y deliberado mediante el cual se pretende alterar determinados factores de la empresa (Benavides, 1998:77).

Se le concede demasiado énfasis a la innovación tecnológica, en ocasiones en detrimento del desarrollo comercial. Como resultado, bastantes tecnologías viables no son comercializadas por la ausencia de marketing o de habilidades empresariales adecuadas (Udell, 1988:62).
La innovación no se debe limitar simplemente a un término técnico, sino que debe entenderse como un fenómeno económico, social, organizativo, estratégico (Fernández et al, 1999a: 239) con referencia a los cambios en los procedimientos establecidos de hacer las cosas.

El proceso que da lugar a la innovación requiere un conocimiento especializado, organización y soporte financiero, por lo que la mayor parte del gasto en investigación y desarrollo (I+D) se da en las grandes empresas.

En las últimas décadas se ha producido un importante incremento de las alianzas entre grandes compañías en todo el mundo y, en menor medida, también se produce en la pyme.

Se señala, en términos generales, que existen tres tipos de cooperación empresarial (Martín y Gaspar, 2005):

a) Cooperaciones horizontales: aquellas que se realizan entre empresas que se dedican a la misma actividad, siendo competidores directos, actuales o potenciales. Suelen perseguir el incremento de su poder frente al resto de competidores, clientes y proveedores.

b) Cooperaciones verticales: acuerdos entre empresas de distintos niveles de la línea de producción y que generan cambios substanciales en las relaciones entre clientes y proveedores. Se crean sinergias a través de la complementariedad de las actividades de los socios con la finalidad de reducir costos, mejorar la calidad del producto o servicio o reducir la incertidumbre de la logística. c) Cooperaciones simbióticas: cooperaciones entre empresas que no tienen ninguna relación entre sí, pero poseen capacidades y competencias complementarias para la realización de un proyecto concreto. 
El capital organizativo de una empresa incluye los acuerdos de cooperación que tiene con proveedores, distribuidores y socios tecnológicos, además de su entramado de rutinas organizativas y su cultura empresarial. Estos elementos pueden considerarse como mecanismos de vinculación y movilización de los distintos factores productivos empleados, que facilitan la conversión de los inputs en bienes y servicios, creando valor al tiempo que determinan la eficiencia y la propia capacidad de innovación de la empresa (Fernández et al, 1998:88).

La cooperación entre empresas se fundamenta en dos pilares:

1. la globalización de la economía, que ha derivado en un entorno general en el que a la empresa le resulta cada vez más difícil afrontar los nuevos desafíos en solitario dada la extensión de la competencia a un nivel mundial, la dispersión de los recursos necesarios para afrontar el crecimiento, el desconocimiento de las peculiaridades político-legales y/o culturales de los nuevos mercados, entre otros asepectos (Urra, 1999:37).

La globalización de los mercados parece haber sido el principal factor que ha impulsado las alianzas estratégicas, reduciendo la eficacia de los planteamientos sencillos y provocando que las empresas deban innovar continuamente para ir por delante de sus competidores. En este sentido, las alianzas se han convertido en un instrumento que está permitiendo a las empresas remodelar sus estrategias competitivas, en respuesta a la globalización (GarcíaOchoa, 2002:139).

2. Niveles de innovación tecnológica crecientes, con una mayor velocidad en la difusión y extensión mundial de las innovaciones, las empresas de reducido tamaño, se ven obligadas a unir sus fuerzas y conseguir alianzas que permitan una acumulación de capitales capaces de afrontar esfuerzos en investigación, desarrollo e innovación (I+D+i).

En este punto, en lo que hace referencia a la relación del tamaño empresarial con la participación de pymes en proyectos de cooperación (y no únicamente para investigación), la teoría no ofrece una guía clara, pues cabría sostener tanto que las pequeñas empresas tienen mayor necesidad de acuerdos cooperativos (dados sus menores recursos internos), como sostener que las empresas grandes tienen mayores recursos internos y se embarcan en un número más amplio de actividades, es probable que puedan beneficiarse de más oportunidades de cooperar (Navarro, 2002: 54).

La cooperación ofrece a las pequeñas y medianas empresas un enorme potencial como consecuencia de la unión de sus capacidades individuales que les permite competir, desde posiciones mejores, con las grandes empresas y hacer frente al incremento de la competencia nacional e internacional.

Lo cierto es que, tanto pymes como grandes empresas, deben asumir que de manera individualista difícilmente conseguirán mantener una posición de liderazgo en el mercado (Martín y Gaspar , 2005:33) y, si bien en un principio las alianzas eran excepciones a las operaciones normales, puesto que eran formadas en un contexto "ad hoc" para tratar situaciones específicas generalmente ligadas al acceso a productos y el control de mercado, el cambio significativo que el entorno ha experimentado ha ido variando esta concepción del fenómeno. 
Las pequeñas empresas pueden competir cuando se organizan de una forma adecuada. $Y$ esta competencia no tiene que tener lugar necesariamente en una situación de condiciones de trabajo y remuneración inferiores ${ }^{3}$.

El principio de asimetría de Joseph Steindl (1945), establece que todo lo que puede ser hecho por una pequeña empresa, puede ser hecho por una gran empresa, pero no a la inversa.

Si ciertas economías en costos están disponibles para instalaciones de una cierta dimensión, tan solo aquellas empresas suficientemente grandes para poder permitirse la inversión de capital que exige dicha instalación se podrán apropiar de esas economías; todas las empresas de dimensiones menores (y las dimensiones se miden aquí por el capital) no podrán.

Por otro lado, si existen economías accesibles para instalaciones pequeñas (el desarrollo técnico puede quizá favorecer a las instalaciones de pequeñas dimensiones) toda empresa mayor puede valerse exactamente como una pequeña empresa, porque nada le impide invertir en distintas pequeñas instalaciones (Steindl, 1945:45).

Sería inmediata la conclusión de que las pequeñas empresas sólo pueden sobrevivir en los rincones muertos del mercado, donde las grandes empresas no tienen interés en entrar pero hay fórmulas organizativas de la producción, entre ellas el distrito industrial, que desmienten aquel principio (Becattini, 2006:24).

Asimismo, la cooperación genera un efecto de "eficiencia colectiva", según la cual las pequeñas empresas comparten y se asocian para superar las desventajas de su reducido tamaño mediante la explotación de las fuerzas innovadoras (flexibilidad, velocidad) derivadas de dicho tamaño.

Esta cooperación, aparece cuando el tamaño de la empresa se convierte en un obstáculo para el crecimiento y la pyme se halla frente a serias dificultades para abordar en solitario los retos que plantea el entorno.

Cuando un empresario se plantea la opción de cooperar con otra empresa, tendrá en cuenta criterios como la compatibilidad estratégica y organizativa, el grado de complementariedad entre las actividades desarrolladas por cada parte, la inter-dependencia mutua que puedan surgir, la confianza así como el grado de compromiso que adquirirán para valorar a su socio potencial (Mora, 2002:54).

La cooperación a través del asociacionismo, es la herramienta que realmente potencia, cuando no genera, un salto cualitativo en el ciclo de vida de una pyme y además es el instrumento que puede ayudar a completar los objetivos estratégicos de una empresa: asociarse para innovar, para reducir costos variables o repartir costos fijos, para obtener mejores condiciones de compra, entre otros.

\section{3.- Redes para la cooperación}

Las redes consisten en una forma de cooperación entre organizaciones en la que, mediante el establecimiento de unos lazos relacionales (relaciones administrativas) éstas se implican

3 Se ha comprobado la concurrencia, para un mismo sector, de talleres de trabajo intenso con escasa remuneración junto a pequeñas empresas con remuneraciones relativamente altas y que utilizan la última tecnología (Pyke y Sengenberger, 1993:208). 
en la realización de un proyecto empresarial.

Se caracterizan por la existencia de una pluralidad de acuerdos entre diversos participantes, bien sean empresas o entre éstas y otro tipo de instituciones tanto públicas como privadas (Fernández et al, 1999b:47).

Una red de empresas es la estructura mínima que mantiene unidas a un grupo de empresas inconexas, proporcionando a éstas todos los beneficios y ventajas de la acción conjunta.

Cada red es una célula. A partir de la creación sistemática de dichas células en un sector -generalmente industriales posible construir una porción de tejido, el cual si es apoyado por los agentes económicos locales a través de instrumentos específicos (como las Escuelas de Empresas) puede transformarse en un distrito ${ }^{4}$.

Desde este punto de vista, el modelo del distrito industrial o clúster es producto del trabajo sistemático hacia adentro y hacia fuera. El trabajo hacia dentro estaría definido por la construcción sistemática de células en la forma de redes empresariales, en tanto que el trabajo hacia fuera se definiría por el nivel de participación de los otros agentes económicos locales proporcionando instrumentos de apoyo (López Cerdán, 1999:17).

Freeman (1991:502), enumera hasta diez tipos $^{5}$ distintos de redes de innovación según su alcance tecnológico, mientras que otros autores como Cooke y Morgan (1994:115) distinguen sólo dos tipos de redes (Inter-empresariales e Intra-empresariales).

Asimismo, se diferencia entre redes que se definen por su mayor integración posible de la I+D, con existencia de elevados estándares de calidad a costos defendibles y una fuerte descentralización de las decisiones de producción, y las que se caracterizan por una relación estrecha y a largo plazo entre productores y usuarios, la presencia de efectos de aprendizaje, la obtención de ventajas de especialización y coordinación, así como por una subcontratación cooperativa a largo plazo para fomentar la innovación tecnológica.

Más homogeneidad se encuentra a la hora de analizar sus características típicas:

- Redundancia: relativamente baja dependencia de los socios frente a la red. Son autónomos, participan voluntariamente en la red, disponen de alternativas y pueden optar por otras redes u otras formas de organización económica. Sin embargo, existe un componente de identificación con el grupo, que determina su propensión a la cooperación (Milton y Westphal, 2005:193).

- La falta de especificidad de las relaciones de intercambio, que no están exactamente definidas.

4 El sector donde aparece este fenómeno con mayor claridad es en el industrial, si bien también aparecen con fuerza en el sector tecnológico (distritos industriales y distritos tecnológicos).

5 a) Joint ventures y proyectos de investigación y acuerdos mutuos en I+D, b) Acuerdos para el intercambio de tecnologías, c) Inversiones directas inducidas por la tecnología (participaciones minoritarias), d) Acuerdos de licencia y second sourcing, e) Subcontratas, f) Divisiones de producción y redes de proveedores, g) Colectivos de investigación, h) Proyectos de investigación impulsados por la administración pública, i) Bancos de datos electrónicos, j) Redes para el intercambio tecnológico y científico orientadas hacia la cadena de valor y otras redes, incluidas las informales. 
- La ausencia de relaciones jerárquicas o, en su caso, la baja significación de las mismas, lo que permite a los socios de la red cooperar en igualdad, si bien en competencia.

- La reciprocidad de las relaciones, que entre los socios de la red puede fluir en ambos sentidos.

- Relaciones a largo plazo en la mayoría de los casos.

- Una alta flexibilidad, dada la falta de vínculos contractuales, ya que, según la necesidad de recursos, es posible permitir la entrada a nuevos miembros o, en su caso, la salida de los socios originales.

- Mayor agilidad y riesgos de burocratización menores, ya que al estar compuesto por pequeñas firmas, hay una ausencia de vínculos contractuales (Mahmood y Rufin, 2005:340).

- Economías de escala gracias al acceso a recursos externos.

- Generación de confianza: al favorecerse la circulación de la información y eliminar comportamientos oportunistas, evitando engaños o fraudes en los negocios y produciendo consecuencias económicas que favorecen los intercambios entre empresas.

Las redes, suelen estar vinculadas a las ventajas de localización, sobre todo en el campo de las altas tecnologías, donde sólo se podrán desarrollar redes allí donde existan ventajas de emplazamiento para determinados sectores (por ejemplo, gracias a la presencia de determinados suministradores de productos y servicios), de ahí que las redes de innovación no se desarrollen generalmente de forma dispersa, sino que presenten una concentración regional.

El profesor Dini (1997), en su estudio de los distritos industriales, señala que las redes de empresas se diferencian de los distritos industriales por las siguientes razones:

1. Las redes de empresas están generalmente constituidas por un número mucho más limitado de firmas que los distritos industriales.

2. Las empresas que componen una red son claramente identificabas y la composición de la misma tiende a ser menos variable.

3. Finalmente los miembros de una misma red no pertenecen necesariamente al mismo territorio.

\section{De las redes de empresas a la aglomeraciones empresariales}

Se pueden diferenciar cinco grandes enfoques teóricos sobre las aglomeraciones empresariales:

1.Nueva Geografía Económica: Elaborada a partir de las contribuciones pioneras de A. Marshall. Las aglomeraciones resultan de la acción acumulativa inducida por la presencia de economías externas locales. Las economías externas son incidentales, y la estructura espacial de la economía es determinada por procesos de mano invisible que opera fuerzas centrifugas y centrípetas. Poco espacio para políticas públicas. (Krugman, 1998:23)

2. Economía de los negocios: Enfatiza la importancia de economías externas geográficamente restrictivas (concentraciones de habilidades y conocimientos altamente especializados, instituciones, rivales, actividades correlacionadas y consumidores sofisticados) en la competencia internacional y en el concepto de clúster. Las estrategias locales nacionales son parte de 
la estrategia de los negocios. Las fuerzas de los mercados determinan el desempeño de los clúster. El gobierno debe proveer educación, infraestructura física y reglas para la competencia. (Porter, 1998)

3. Economía Regional: para Scott (2000), geografía económica y desempeño industrial están interrelacionados. Existe una tendencia endémica en el capitalismo en dirección a compactos clústers localizados. Estos son constituidos como economías regionales intensivas en transacciones que a su vez son enlazadas por estructuras de interdependencia que están dispersas mundialmente. Coordinación extra mercado y políticas públicas son esenciales en la construcción de ventajas competitivas localizadas. (Scott, 2000)

4. Economía de la innovación: La proximidad local facilita el flujo de información y la diseminación del conocimiento. Actividades económicas con base en nuevos conocimientos tienen una gran propensión a agruparse dentro de regiones geográficas (Audretsch et al, 2004).

5.Pequeñas empresas y distritos industriales: Además de las economías externas locales incidentales o espontáneas, existe también una fuerza deliberada en acción, derivada de la cooperación consistente entre agentes privados y del apoyo del sector público. El concepto de eficiencia colectiva combina los efectos espontáneos (o no planificados) y aquellos conscientemente buscados (o planificados), y es definida como la ventaja competitiva derivada de las economías externas locales y de la acción conjunta (Schmitz, 1992).

Para el profesor Becattini (2006) todo comienza con Marshall (1919) y con su planteamiento de que existen dos tipos de producciones eficientes. Las basadas en grandes unidades productivas, eminentemente verticales (paradigma fordista) y la concentración de numerosas fábricas de pequeñas dimensiones y especializadas en diferentes fases de un único proceso colectivo en una o varias localidades.

Para este autor, la economía del distrito ${ }^{6}$, como de las empresas individuales del distrito, se caracteriza por la continua valoración del beneficio diferencial de los costes de producción entre el interior y el exterior.

En este argumento, Becattini (2006) admite de forma implícita que el empresario realice constantemente un cálculo económico, de tipo marginalista, para valorar las ventajas y los inconvenientes con el pretexto de establecer, de seguir y de aumentar sus actividades industriales sobre el propio terreno (Saglio, 1993: 130).

Se puede afirmar que mediante el estudio del distrito, economía y geografía han encontrado un terreno de encuentro

6 Autores como Becattini (2006), Bellandi (2006) o Sforzi (2006) entre otros, a los cuales directa o indirectamente se hace referencia en esta tesis, han investigado en la experiencia italiana de distrito industrial (regiones como Toscana, Veneto o Emilia-Romagna), siendo cuna y madre de este concepto. Esta línea de pensamiento fue rápidamente seguida (1993) por otros autores como Quintar, Ascua o Gatto, encontrando similitudes en regiones argentinas denominándolas "cuasi-distrito italiano". 
como tal vez nunca antes tuvieron a disposición, ni siquiera con la economía regional (Sforzi, 2006: 40).

Actualmente, los trabajos sobre localización industrial (Alañón et al, 2006: 2) pueden agruparse en tres escuelas:

1. La aproximación neoclásica, es aquella que está más relacionado con la teoría de la localización clásica y centra su análisis en la maximización de beneficios y en las estrategias de minimización de costos.

2. La aproximación de conducta, trata de las decisiones sobre localización que se producen en un contexto de información imperfecta y de incertidumbre. A diferencia de la aproximación neoclásica, el proceso de toma de decisión del empresario está basado también en factores no estrictamente económicos.

Finalmente, la aproximación institucional, mantiene que en el proceso de localización es importante considerar no solamente la empresa que está buscando un emplazamiento, sino también el entorno institucional existente en esa localización.

El estudio del distrito ha tenido un papel decisivo al plantear de una forma nueva el problema del territorio en el ámbito económico, además del geográfico.

Los distritos son un modelo de organización de la producción en el que el papel de las fuerzas sociales locales es importante y se caracteriza por un "deseo de comunidad" en la medida en que insta a competir a las comunidades locales en su conjunto (Lazzeretti, 2006: 68).

Se consideran claves para la determinación de un distrito industrial: Un sistema productivo localizado geográficamente, factores diversos que determinan el grado de proximidad del modelo real al ideal y la necesaria actuación colectiva que permite al distrito industrial caminar por la vía ancha o ser considerado como "especialización flexible" (Zeitlin, 1993: 366).

A principio de los años 90, con el impulso de una importante investigación conducida por Michael Porter (1998), se abre una vía en la problemática del distrito, especialmente en Italia, observando concentraciones territoriales de pequeñas empresas que han adquirido ventajas competitivas. Porter insiste en el concepto de clúster, hasta hacerlo muy cercano al de distrito industrial marshaliano.

De manera esquemática, se puede reconocer un distrito industrial por la existencia de los siguientes elementos (Guenzi, 2006: 33):

- En los distritos industriales comunidad y sistema de empresas son elementos indivisibles.

- Existe una particular ética del trabajo y de la profesión, del riesgo y del cambio.

- La sociedad del distrito industrial nace y se desarrolla a través de instituciones y reglas que conservan y transmiten los valores.

- Es una sociedad "abierta", dispuesta a aceptar nuevas formas de iniciativa económica y al mismo tiempo "cerrada", preparada para conminar sanciones sociales a las actividades que no respetan las reglas.

- Puede existir una red estable de distribución de los productos en el extranjero.

- El predominio de producciones y de intercambios relativos a los bienes y a los servicios destinados a la exportación.

- La identidad de los productos vinculada a particulares cualidades (la imagen del producto referida al distrito más que a la empresa). 
- Un sistema de regulación y control de los precios y de las prestaciones y de los semi-elaborados inherentes a la producción típica.

Los distritos industriales son normalmente identificados con sistemas locales de producción (al menos como una de sus posibles formas), que producen productos locales competitivos con formas organizativas similares. Aunque el conjunto de relaciones que se desarrolla en base a la proximidad geográfica pueden variar considerablemente en sus detalles, su lógica fundamental es constante (MolinaMorales, 2005:160).

Son exitosos aquellos que generan una densa red de relaciones de cooperación (horizontales y verticales).

La concentración productiva no hace referencia únicamente a la aglomeración de pequeñas firmas dedicadas a un mismo sector, sino a la desintegración del proceso productivo en diferentes empresas y su reconstitución a través de vínculos ínter empresariales de cooperación vertical.

Es así como la concentración productiva y las relaciones de cooperación permiten la especialización de las empresas, y la conformación de una cadena productiva (desde los proveedores de insumos básicos hasta los agentes de comercialización) altamente fragmentada $y$, al mismo tiempo, fuertemente integrada y cohesionada dentro del clúster.

Por otra parte, la concentración productiva favorece el establecimiento de relaciones horizontales que permiten ganar fuerza a empresas pequeñas y obtener beneficios colectivos que difícilmente les serían accesibles en forma individual (Bueno y Saraví, 1997:108).

En el análisis del efecto distrito, las variables clave, así como la atmósfera social y los flujos de información del entorno, son difíciles de medir y, además, los procesos causales no son directamente observables.

Para detectar el efecto distrito se deben analizar las características del modelo de localización industrial, incluyendo variables externas. Las aglomeraciones que supongan una especialización sectorial conducirán directamente a las precondiciones para la existencia de un distrito industrial y de externalidades marshallianas.

Las que supongan diversidad sectorial pueden generar otro tipo de externalidades, las de urbanización, que no están contempladas en el efecto distrito (Elizalde, 2003:31).

El ambiente típico del distrito constituye una especie de incubadora de capacidades empresariales. La difusión de estas capacidades es favorecida por la distribución del trabajo, que baja los costeo de entrada, mientras el clima de confianza recíproca reduce los riesgos del emprendedor (empresario) aspirante.

La difusión de las capacidades emprendedoras es un elemento crucial en cuanto que, sobre todo a través del surgimiento de nuevas empresas especializadas, el distrito como sistema puede cambiar, manteniendo la propia.

Ahora bien, este elemento que también hace referencia a la prestación de servicios por parte de las instituciones colectivas y a la resolución de los conflictos entre los agentes, puede entenderse desde un punto de vista estratégico como resultado de la gestión de los recursos compartidos existentes en los distritos.

Las externalidades de las que se benefician las empresas, y que se concretan en los servicios comunes, son consecuencia de los flujos de información $y$ de conocimientos que existen en el distrito industrial y que tienen su soporte 
organizativo en la propia red que éste constituye.

De tal manera que esta dotación de recursos compartidos se puede vincular con la ventaja competitiva de las empresas miembros. La existencia de los recursos compartidos es la que justifica la ejecución de una estrategia colectiva monitorizada por las instituciones colectivas.

Es dentro de este proceso estratégico donde se deben enmarcar la resolución de los conflictos entre los participantes. El ámbito que suponen los recursos compartidos es dominante respecto al ámbito de los recursos exclusivos de una organización. Así su capacidad competitiva se encuentra ligada al distrito industrial en su conjunto (Camison y Molina, 1998:19).

En cuanto a los retos y dificultades que los distritos industriales se encontraran a medio y largo plazo, Bellandi (2006:44), destaca el desafío de las nuevas industrias en la incorporación de las nuevas tecnologías de la información y de la comunicación por parte de las multinacionales, el desafío de las nuevas industrias (China, India, Brasil...), y una tendencia neo-artesanal.

\section{Conclusiones}

La innovación empresarial se debe entender como transformación de conocimiento en nuevos productos, servicios o procesos, o bien, en cambios significativos y diferenciales en los existentes, con el objetivo de introducirlos en el mercado y conseguir una ventaja competitiva. Al ser un recurso intangible tiene una significativa influencia en la competitividad empresarial debido a que es realmente el factor que marca la diferencia entre empresas en un mercado globalizado. Las empresas tienen facilidad de acceso a las fuentes de información y conocimiento, y por lo tanto de innovar. La eficiencia dependerá de la utilización más racional de este recurso.

Asimismo, se puede afirmar que mediante la combinación de la cooperación estratégica e innovación se logra que pequeñas y medianas empresas tengan potencial suficiente para competir con las grandes compañías desde mejores posiciones, permitiendo concentraciones de empresas y promoviendo acciones de desarrollo local.

Teniendo muy presente el principio de asimetría de Steindl y el fenómeno de la globalización, el futuro de la pequeña empresa sería cuanto menos incierto, si no fuera por la oportunidad de introducirse más eficazmente en el mercado mediante concentraciones de empresas (redes de cooperación y distritos industriales).

Las externalidades de las que se benefician las empresas, y que se concretan en los servicios comunes, son consecuencia de los flujos de información $y$ de conocimientos que existen en el distrito industrial y que tienen su soporte organizativo en la propia red que éste constituye.

Por último, se puede asegurar que el efecto llamado propicia la secuencia del círculo virtuoso: Red Empresarial - Cooperación - Innovación - Red empresarial. Estas son las variables y pasos que necesariamente debe de llevar a cabo una pyme para poder competir en un mercado cada vez más globalizado. 


\section{Referencias Bibliográficas}

Alañón, Ángel y Arauzo, José María (2006), Accesibilidad y localización industrial. Una aplicación para las Comunidades Autónomas fronterizas con Francia. Documentos de trabajo de la Facultad de Ciencias Económicas y Empresariales 06-01. Universidad Complutense de Madrid.

Audrechts, David Bruce y Feldmann, Morton (2004), Knowledge spillovers and the geography of innovation en J.V. Henderson y J.F. Thisse eds. Handbook of Urban and Regional economics, Vol. 4, Noth-Holland.

Becattini, Giacomo (2006), Vicisitudes y potencialidades de un concepto: El distrito industrial. Revista de Economía Industrial $n^{\circ} 359$, pp. 21-27.

Bellandi, Marco (2006), El distrito industrial y la economía industrial. Algunas reflexiones sobre su relación. Revista de Economía Industrial $n^{\circ} 359, p p$. 43-57.

Benavides, Carlos (1998), Tecnología, innovación y empresa. Ediciones Pirámide.

Bueno, Carmen y Saraví, Gonzalo. (1997), Relaciones de cooperación interempresarial. Lecciones de la experiencia Mejicana. Revista Nueva Sociedad, $\mathrm{n}^{\circ}$ 151. SeptiembreOctubre, pp. 102-121.

Camison, César y Molina, Frances Xavier (1998), Evaluación de la proximidad de una colectividad de organizaciones al modelo ideal de distrito industrial y desempeño empresarial: una aplicación a los casos de los distritos de la industria cerámica de Italia y España. Revista de Estudios Regionales, $n^{\circ}$ 50, pp. 15-37.

Caravaca, Inmaculada, González, Gema y Silva, Rocío (2003), Redes e Innovación socio-institucional en sistemas productivos locales. Boletín de la A.G.E. n 36. pp. 103-115.

Cooke, Philip y Morgan, Kevin (1994), Growth regions under duress: renewal strategies in Baden-Wurtemberg and Emilia-Romagna. Amin \& Thrift, pp. 91-117.

Dini, Marco (1997), Enfoques conceptuales para el estudio de pequeñas y medianas empresas, Mimeo, Santiago de Chile: CEPAL

Elizalde, Antonio (2003), Planificación estratégica territorial y políticas públicas para el desarrollo local. Instituto Latinoamericano y del Caribe de Planificación Económica y Social (ILPES). Serie Gestión Pública.

Fernández, Javier (2002), Crear empresa: Mil consejos para un emprendedor. Cuarta Edición. Cie Inversiones Editoriales Dossat 2000.

Fernández, Juan Carlos y Arranz, Nieves (1999a), Las redes de cooperación ¿una organización para el nuevo milenio?. Revista Cepade $\mathrm{n}^{\circ} 2$, pp. 12-19.

Fernández, Juan Carlos y Arranz, Nieves (1999b), La cooperación entre empresas. Análisis y diseño. ESIC Editorial.

Fernández, Esteban; Montes, José Manuel y Vázquez, Camilo José (1998), Los Recursos Intangibles como Factores de Competitividad de la Empresa. Revista Dirección y Organización $n^{\circ}$ 22. Septiembre, pp. 83-98.

Freeman, Chris (1991), Networks of innovators: a synthesis of research issues. Research Policy, 20, pp. 499514

Frías, José (2006), La Tercera Edición del Manual de Oslo amplía el concepto de Innovación a la de carácter no Tecnológico. Revista Economía Industrial $n^{\circ} 360$, pp. $217-228$

García-Ochoa, Mónica (2002), Estudio Empírico sobre la cooperación empresarial en España. Revista CEPADE n 27 , pp. 139-147.

Gisbert, Santiago. (2005), Creatividad e innovación en la práctica empresarial. Fundación COTEC para la Innovación Tecnológica. 
Guenzi, Alberto (2006), El distrito industrial y su impacto en la Historia Económica. Revista de Economía Industrial $\mathrm{n}^{\circ}$ 359. pp. 29-36.

Hidalgo, Antonio; León, Gonzalo y Pavón, Julián. (2002), La gestión de la innovación y la tecnología en las organizaciones. Ediciones Pirámide.

Krugman, Paul (1998), What's new about the new economic geography?, Oxford Review of Economic Policy, Vol. 14, No 2

Lapiedra, Ramón. (1999), El desarrollo de alianzas: Una solución estratégica para PYMES. Revista CEPADE $\mathrm{n}^{\circ}$ 21. Enero, pp. 161-166.

Lazzerattl, Luciana (2006), Distritos industriales clústers y otros: un análisis trespassing entre la economía industrial y la gestión estratégica. Revista de Economía Industrial $\mathrm{n}^{\circ}$ 359, pp. 59-72.

López-Cerdán, Carlos (1999), Distritos industriales: Experiencias de acción conjunta y cooperación interempresarial para el desarrollo de la pequeña y mediana industria. Espacios. Vol. 20 (2).

Lundvall, Bengt-Ake, (1992), National systems of innovation, London: Pinter.

Mahmood, Ishtiaq P. y Rufin, Carlos (2005), Government's dilema: The role of government in imitation and innovation. Academy of Management Review. Vol. 30, n 2, pp. 338-360.

Martín, Inmaculada y Gaspar, Ana (2005), La cooperación empresarial como estratega para lograr sinergias pymes y grandes empresas: Estudio empírico sobre el sector turístico español. Boletín Económico de ICE $n^{\circ} 2844$ Mayo, pp. 25-36.

Marshall, Alfred (1919), Industry anda Trade. Macmillan

Milton, Lauri P. Y Westphal, James D. (2005), Identity confirmation networks and cooperation in work groups.
Academy of Management Journal. Vol. 48, n 2 , pp. 191-212.

Molina-Morales, Francisco (2005), Estrategias de exploración y explotación en las aglomeraciones territoriales de empresas: Una aproximación desde la perspectiva del capital social. Revista Valenciana de Economía y Hacienda $\mathrm{n}^{\circ}$ 13, pp. 157-184.

Mora, Eva María (2002), Cooperación entre empresas versus cooperación Universidad-empresa: criterios para la selección de socios en acuerdos de cooperación tecnológica. Revista CEPADE n ${ }^{\circ} 27$. Febrero, pp. 44-56.

Morcillo, Patricio (2007), Cultura e Innovación Empresarial. La conexión perfecta. Thomson Editores Spain.

Navarro, Mikel (2002), La cooperación para la innovación en la empresa española desde una perspectiva internacional comparada. Revista de Economía Industrial $n^{\circ} 346$, IV, pp. 47-66.

Navas, José E. y Guerras, Luís A. (1998), La Dirección Estratégica de la Empresa. Teoría y Aplicaciones. Cívitas Segunda edición.

Nelson, Richard R. (1993), National innovation systems: a comparative analysis, New York: Oxford University Press.

Ondategui, Julio César (2001), Los Parques Científicos y Tecnológicos en España: Retos y Oportunidades. Dirección General de Investigación de la Comunidad de Madrid.

Organisation for Economic Co-Operation and Development (2005), Oslo Manual: Guidelines for Collecting and Interpreting Innovation Data, 3rd edition. OECD PUBLICATIONS.

Pyke, Frank y Sengenberger, Werner. (1993), Los distritos industriales y las pequeñas empresas. Colección Economía y Sociología del Trabajo. Vol III. Ministerio de Trabajo y Seguridad Social.

Porter, Michael (1998), The Competitive advantage of Nations: with a new 
introduction, MacMillan, Reino Unido.

Rodríguez-Pose, Andrés (1999), Innovation prone, and innovation adverse societies: Economics performance in Europe. Growth and Change. Vol 30 (Winter), pp. 75-105.

Saglio, Jean (1993), Concepto de la empresa, elección del empresario y cambio social. Consideraciones para una teoría de sociología económica. Revista Política y Sociedad $n^{\circ} 13$, pp. 125-139.

Sainz de Vicuña, José María (2006), Innovar con éxito. ESIC Editorial.

Schmitz, Hubert (1992), Industrial districts: model and reality in BadenWurtemberg, Germany. En: Pyke, F. \& Segenberger, W. comps. Industrial districts and local economic regeneration. Ginebra: IILS, pp. 87121.

Schumpeter, Joseph A. (1939), Business Cycles: A theoretical, historical and statistical analysis of the Capitalist process. McGraw-Hill.

Scott, Allen J. (2000), Economic geography: the great half-century, Cambridge Journal of Economics, Vol. 24, pp. 483-504.

Sforzi, Fabio (2002), Sistemas Locales de empresa y transformación industrial en Italia, Manual de Desarrollo Local de Fermin Rodriguez Gutierrez. Ed. Trea.

Sforzi, Fabio (2006), El distrito industrial y el «viraje territorial» en el análisis del cambio económico. Revista de Economía Industrial $n^{\circ} 359$, pp. $37-$ 42.

Steindl, Josef (1945), Small and Big Business. Economic Problems of the Size of Firms. Blackwell.

Udell, Gregory (1988), Strategies for Stimulating Home-grown TechnologyBased Economic Development. Business Horizons. NoviembreDiciembre, pp. 60-64.

Urra, José Anastasio (1999), Cooperación Interempresarial. Algunas puntualizaciones en una aproximación económica al fenómeno. Revista CEPADE $n^{\circ} 22$, pp. 37-49.

Zeitlin, Jonathan (1993), Distritos industriales y regeneración económica local: Visión general y comentarios, en F. Pyke y W. Sengenberger compiladores: Los distritos industriales y las pequeñas empresas. Colección Economía y Sociología del Trabajo. Vol. III, Ministerio de Trabajo y Seguridad Social, pp. 359-378. 